\title{
A criterion for unimodality
}

\author{
George Boros \\ Department of Mathematics, University of New Orleans \\ New Orleans, LA 70148 \\ gboros@math.uno.edu \\ Victor H. Moll ${ }^{1}$ \\ Department of Mathematics, Tulane University \\ New Orleans, LA 70118 \\ vhm@math.tulane.edu \\ Submitted: January 23, 1999; Accepted February 2, 1999 \\ Classification 05, 33, 40
}

\begin{abstract}
We show that if $P(x)$ is a polynomial with nondecreasing, nonnegative coefficients, then the coefficient sequence of $P(x+1)$ is unimodal. Applications are given.

\section{INTRODUCTION}

A finite sequence of real numbers $\left\{d_{0}, d_{1}, \cdots, d_{m}\right\}$ is said to be unimodal if there exists an index $0 \leq m^{*} \leq m$, called the mode of the sequence, such that $d_{j}$ increases up to $j=m^{*}$ and decreases from then on, that is, $d_{0} \leq d_{1} \leq \cdots \leq d_{m^{*}}$ and $d_{m^{*}} \geq d_{m^{*}+1} \geq \cdots \geq d_{m}$. A polynomial is said to be unimodal if its sequence of coefficients is unimodal.

Unimodal polynomials arise often in combinatorics, geometry and algebra. The reader is referred to [2] and [3] for surveys of the diverse techniques employed to prove that specific families of polynomials are unimodal.

A sequence of positive real numbers $\left\{d_{0}, d_{1}, \cdots, d_{m}\right\}$ is said to be logarithmically concave (or log-concave for short) if $d_{j+1} d_{j-1} \leq d_{j}^{2}$ for $1 \leq j \leq m-1$. It is easy to see that if a sequence is log-concave then it is unimodal [4]. A sufficient condition for log-concavity of a polynomial is given by the location of its zeros: if all the zeros of a polynomial are real and negative, then it is log-concave and therefore unimodal [4]. A second criterion for the log-concavity of a polynomial was determined by Brenti [2]. A sequence of real numbers is said to have no internal zeros if whenever $d_{i}, d_{k} \neq 0$ and $i<j<k$ then $d_{j} \neq 0$. Brenti's criterion states that if $P(x)$ is a log-concave polynomial with nonnegative coefficients and with no internal zeros, then $P(x+1)$ is log-concave.
\end{abstract}

\footnotetext{
${ }^{1}$ www: <http: //www.math.tulane.edu:80/ vhm>
} 
THe EleCtronic Journal of COMbinatorics 6 (1999), \#R10

\section{The MAIn Result}

Theorem 2.1. If $P(x)$ is a polynomial with positive nondecreasing coefficients, then $P(x+1)$ is unimodal.

Proof. Observe first that $P_{m, r}(x):=(1+x)^{m+1}-(1+x)^{r}$ with $0 \leq r \leq m$ is unimodal with mode at $1+\left\lfloor\frac{m}{2}\right\rfloor$. This follows by induction on $m \geq r$ using $P_{m+1, r}(x)=P_{m, r}(x)+x(1+x)^{m+1}$. For $m$ even, $P_{m+1, r}$ is the sum of two unimodal polynomials with the same mode. For $m=2 t+1$, the modes are shifted by 1 , so it suffices to check

$$
a_{t+1}+\left(\begin{array}{c}
m+1 \\
t
\end{array}\right) \leq a_{t+2}+\left(\begin{array}{c}
m+1 \\
t+1
\end{array}\right)
$$

where $a_{t+1}$ is the coefficient of $x^{t}$ in $P_{m, r}(x)$. The case $t \geq r$ yields equality in $(2.1)$. If $t \leq r-2$ then (2.1) is equivalent to $r \leq m+2$. The final case $t=r-1$ amounts to $0=\left(\begin{array}{c}m+1 \\ r-1\end{array}\right)-\left(\begin{array}{c}m+1 \\ r+1\end{array}\right) \leq 1$,

Now $P(x+1)=\frac{1}{x}\left(b_{0} P_{m, 0}(x)+\left(b_{1}-b_{0}\right) P_{m, 1}(x)+\cdots+\left(b_{m}-b_{m-1}\right) P_{m, m}(x)\right)$, so $P(x+1)$ is a sum of unimodal polynomials with the same mode, and hence unimodal.

We now restate Theorem 2.1 and offer an alternative proof.

Theorem 2.2. Let $b_{k}>0$ be a nondecreasing sequence. Then the sequence

$$
c_{j}:=\sum_{k=j}^{m} b_{k}\left(\begin{array}{l}
k \\
j
\end{array}\right), \quad 0 \leq j \leq m
$$

is unimodal with mode $m^{*}:=\left\lfloor\frac{m-1}{2}\right\rfloor$.

Proof. For $0 \leq j \leq m-1$ we have

$$
(j+1)\left(c_{j+1}-c_{j}\right)=\sum_{k=j}^{m} b_{k}\left(\begin{array}{l}
k \\
j
\end{array}\right) \times(k-2 j-1) .
$$

Suppose first that $j \geq m^{*}$, and let $m$ be odd so that $m=2 m^{*}+1$; the case $m$ even is treated in a similar fashion. Every term in (2.3) is negative because, if $j>m^{*}$, then $k-2 j-1 \leq m-2 j-1=2\left(m^{*}-j\right)<0$, and for $j=m^{*}$,

$$
\left(m^{*}+1\right)\left(c_{m^{*}+1}-c_{m^{*}}\right)=\sum_{k=m^{*}}^{m-1} b_{k}\left(\begin{array}{c}
k \\
m^{*}
\end{array}\right) \times(k-m)<0 .
$$

Thus $c_{j+1}<c_{j}$.

Now suppose $0 \leq j<m^{*}$ and define

$$
T_{1}:=\sum_{k=j}^{2 j} b_{k}\left(\begin{array}{l}
k \\
j
\end{array}\right)(2 j+1-k)
$$


THE ELECTRONIC JOURNAL OF COMBINATORICS 6 (1999), \#R10

and

$$
T_{2}:=\sum_{k=2 j+2}^{m} b_{k}\left(\begin{array}{l}
k \\
j
\end{array}\right)(k-2 j-1)
$$

so that $(j+1)\left(c_{j+1}-c_{j}\right)=T_{2}-T_{1}$. Then

$$
T_{1}<b_{2 j+2} \sum_{k=j}^{2 j}\left(\begin{array}{c}
k \\
j
\end{array}\right)(2 j+1-k)=b_{2 j+2}\left(\begin{array}{c}
2 j+2 \\
j
\end{array}\right)<T_{2} .
$$

Thus $c_{j+1}>c_{j}$.

\section{Examples}

Example 1. The case $P(x)=x^{n}$ in Theorem 2.1 gives the unimodality of the binomial coefficients.

Example 2. For $0 \leq k \leq m-1$, define

$$
b_{k}(m):=2^{-2 m+k}\left(\begin{array}{c}
2 m-2 k \\
m-k
\end{array}\right)\left(\begin{array}{c}
m+k \\
m
\end{array}\right)(a+1)^{k}
$$

for $0 \leq k \leq m-1$. Then

$$
\frac{b_{k+1}(m)}{b_{k}(m)}=\frac{(m-k)(m+k+1)}{(2 m-2 k-1)(k+1)}>1
$$

so the polynomial

$$
P_{m}(a):=\sum_{k=0}^{m} b_{k}(m)(a+1)^{k}
$$

is unimodal. We encountered $P_{m}$ in the integral formula [1]

$$
\int_{0}^{\infty} \frac{d x}{\left(x^{4}+2 a x^{2}+1\right)^{m+1}}=\frac{\pi P_{m}(a)}{2^{m+3 / 2}(a+1)^{m+1 / 2}} .
$$

This does not appear in the standard tables.

Example 3. For $0 \leq k \leq m-1$, define

$$
b_{k}(m):=\frac{(-m-\beta)_{m}}{m !} \frac{(-m)_{k}(m+1+\alpha+\beta)_{k}}{(\beta+1)_{k} k ! 2^{k}} .
$$

Then, with $\alpha=m+\epsilon_{1}$ and $\beta=-\left(m+\epsilon_{2}\right)$, we have

$$
\frac{b_{k+1}(m)}{b_{k}(m)}=\frac{m-k}{m-k+\epsilon_{2}-1} \times \frac{k-1+m+\epsilon_{1}-\epsilon_{2}}{2(k+1)}>1
$$


The EleCtronic Journal of COMBinatorics 6 (1999), \#R10

provided $0<\epsilon_{1} \leq \epsilon_{2}<1$. Therefore the polynomial

$$
P_{m}^{(\alpha, \beta)}(a):=\sum_{k=0}^{m} b_{k}(m)(a+1)^{k}
$$

is unimodal. This is a special case of the Jacobi family, where the parameters $\alpha$ and $\beta$ are not standard since they depend on $m$. These polynomials do not have real zeros, so their unimodality is not immediate. The case of Example 2 corresponds to $\epsilon_{1}=\epsilon_{2}=\frac{1}{2}$.

Example 4. Let $n, m \in \mathbb{N}$ be fixed. Then the sequences

$$
\alpha_{j}:=\sum_{k=j}^{m} n^{k}\left(\begin{array}{l}
k \\
j
\end{array}\right), \quad \beta_{j}:=\sum_{k=j}^{m} k^{n}\left(\begin{array}{l}
k \\
j
\end{array}\right), \text { and } \gamma_{j}:=\sum_{k=j}^{m} k^{k}\left(\begin{array}{l}
k \\
j
\end{array}\right)
$$

are unimodal for $0 \leq j \leq m$.

Example 5. Let $2<a_{1}<\cdots<a_{p}$ and $n_{1}, \cdots, n_{p}$ be two sequences of $p$ positive integers. For $0 \leq j \leq m$, define

$$
c_{j}:=\sum_{k=j}^{m}\left(\begin{array}{c}
a_{1} m \\
k
\end{array}\right)^{n_{1}}\left(\begin{array}{c}
a_{2} m \\
k
\end{array}\right)^{n_{2}} \ldots\left(\begin{array}{c}
a_{p} m \\
k
\end{array}\right)^{n_{p}}\left(\begin{array}{c}
k \\
j
\end{array}\right) .
$$

Then $c_{j}$ is unimodal.

Acknowledgement. The authors wish to thank Doron Zeilberger for comments on an earlier version of this paper.

\section{REFERENCES}

[1] BOROS, G. - MOLL, V.: An integral hidden in Gradshteyn and Rhyzik, J. Comp. Appl. Math., to appear.

[2] BRENTI, F.: Log-concave and unimodal sequences in Algebra, Combinatorics and Geometry: an update. Contemporary Mathematics, 178, 71-84, 1994.

[3] STANLEY, R.: Log-concave and unimodal sequences in algebra, combinatorics and geometry. Graph theory and its applications: East and West (Jinan, 1986), 500-535, Ann. New York Acad. Sci., 576, New York, 1989.

[4] WILF, H.S.: generatingfunctionology. Academic Press, 1990. 